\title{
On the polarization of the nucleon sea in the meson cloud model
}

\author{
K.G. Boreskov, A.B. Kaidalov \\ ITEP, B.Cheremushkinskaya 25 \\ 117259 Moscow, Russia
}

\begin{abstract}
It is pointed out that the meson cloud model predicts a substantial polarization of the sea quarks of the nucleon due to interference of $\pi$ and $\rho$ exchanges. This polarization is strongly flavour dependent. The model gives an explanation of a strong increase in the structure function $g_{1}$ with isospin $I=1$ at small $x$.
\end{abstract}




\section{Introduction}

Experimental studies of nucleon structure functions have revealed several interesting and unexpected features. Investigation of the Gottfried sum rule by the NMC collaboration [1] lead to the conclusion that $\bar{u}$ and $\bar{d}$ quarks distributions in the proton are different. This result has been confirmed by measurements of the Drell-Yan process [2], [3]. The difference between $\bar{u}$ and $\bar{d}$ distributions is measured now as a function of $x$ [3] and is concentrated in the region of $x \lesssim 0.2$. This phenomenon has a natural explanation [4] - [6] in the meson cloud model (Fig.1) if positive pions in the cloud prevail over negative ones (i.e. the contribution of the diagram of Fig.1a is larger than the one of Fig.1b).

In this paper we want to pay attention to another interesting problem, the unusual small- $x$ behaviour of the spin structure function $g_{1}$ of proton and neutron [7]- 12]. The situation is especially evident in the case of the neutron structure function $g_{1}^{n}$, which is very small in the region of $x \gtrsim 0.2$ and goes to rather large negative values as $x$ decreases to $x \sim 10^{-2}$ [11. This behaviour cannot be explained by valence quark models, first since valence quarks contribute to the function $g_{1}$ mainly at $x \gtrsim 0.1$ and second, because of strong cancellation of valence quark contribution for the neutron 円. At the same time the observed small- $x$ behaviour contradicts to the one expected for the $A_{1}$ Reggepole exchange because of its low intercept. It would be natural to attribute this to the manifestation of the nucleon sea. However, it is usually believed that the meson cloud model cannot lead to a polarization of sea quarks [13], as the diagrams of Fig.1 with $\pi \pi$ exchange in the $t$ channel do not contribute to the function $g_{1}$. In this paper we would like to emphasize that an interference between $\pi$ and $\rho$ exchanges ( $\pi \rho$ exchange in the $t$-channel - Fig.2) leads to a substantial polarization of the nucleon structure function $g_{1}$ with isospin $I=1$ in the $t$-channel. It will be shown also that flavour asymmetry of the polarized quark sea must be even stronger than for the unpolarized case. Experimental tests of this model are proposed.

\footnotetext{
${ }^{1}$ Thus the neutron is a good laboratory for the investigation of non-valence components of the nucleon.
} 


\section{General analysis of the small- $x$ behaviour of the spin-dependent structure functions}

Analysis of the small- $x$ behaviour of structure functions of deep-inelastic scattering on a nucleon in terms of the leading $j$-plane singularities has been performed many years ago [14]. However there is a controversy concerning a possibility of different contributions

to functions $g_{1}(x), g_{2}(x)$ [15]. So we shall repeat here the main consequences of such an analysis.

The polarized structure functions $g_{1}\left(x, Q^{2}\right)$ and $g_{2}\left(x, Q^{2}\right)$ define the antisymmetrical in $\mu, \nu$ part of the amplitude of the virtual Compton scattering (see e.g. [16], [17]):

$$
W_{\mu \nu}^{(A)}(q ; P, S)=\frac{2 m_{N}}{(P \cdot q)} \epsilon_{\mu \nu \rho \sigma} q^{\rho}\left[g_{1}\left(x, Q^{2}\right) S^{\sigma}+g_{2}\left(x, Q^{2}\right) S_{\perp}^{\sigma}\right]
$$

where $q$ is a photon 4 -momentum, $Q^{2}=-q^{2}, P$ and $S$ are nucleon momentum and spin vectors, and

$$
S_{\perp}^{\sigma}=S^{\sigma}-\frac{(S \cdot q) P^{\sigma}}{(P \cdot q)} .
$$

The absorptive parts of the $s$-channel helicity amplitudes $F_{\lambda_{\gamma^{*}}^{\prime} \lambda_{N}^{\prime} ; \lambda_{\gamma^{*}} \lambda_{N}}$ for forward $\gamma^{*} N$ scattering can be written in terms of the functions $g_{1}$ and $g_{2}$ as follows [14]

$$
\begin{aligned}
& \frac{1}{2}\left(F_{1 \pm \frac{1}{2} ; 1 \pm \frac{1}{2}}-F_{-1 \pm \frac{1}{2} ;-1 \pm \frac{1}{2}}\right)= \pm \frac{1}{m_{N}}\left(g_{1}\left(x, Q^{2}\right)-\frac{4 m_{N}^{2}}{Q^{2}} x^{2} g_{2}\left(x, Q^{2}\right)\right) \\
& F_{0 \mp \frac{1}{2} ; \pm 1 \pm \frac{1}{2}}=\frac{\sqrt{2 Q^{2}}}{m_{N} \nu}\left(g_{1}\left(x, Q^{2}\right)+g_{2}\left(x, Q^{2}\right)\right) .
\end{aligned}
$$

The rules for construction of helicity amplitudes with definite $t$-channel quantum numbers are well known (see e.g. [14). In the combination of Eq.(3) of s-channel helicity amplitudes only states with $\sigma P=-1$ and $G(-1)^{I} \sigma=-1$ contribute asymptotically as $x \rightarrow 0$ (here $\sigma$ is the signature, $P$ - parity, $G-G$-parity and $I$ is the isospin). These are the singularities (e.g. Regge poles) of the so called axial group. Among known Regge poles the reggeons $A_{1}(I=1)$ and $f_{1}(I=0)$ belong to this group.

The amplitude (4) corresponds to the states in the $t$-channel, which do not have definite parity (for example Regge -cuts). The rightmost singularity of this type will be 
a cut due to exchange by several Pomerons [2.

Let us note that Regge cuts should also obey the quantum number selection rules. In particular, Regge cuts due to exchange by any number of Pomerons in the $t$-channel do not contribute (in the leading order in $1 / x$ ) to the function $g_{1}$. This is connected to the fact that signature of the cut is equal to a product of signatures of exchanged reggeons 18 and is +1 for any number of exchanged Pomerons. So the product $G(-1)^{I} \sigma$ in this case is +1 and does not correspond to the amplitude $g_{1}$ we are interested in. This result can be generalized to the cuts due to exchange of any number of reggeons with quantum numbers $\sigma P=+1, G(-1)^{I} \sigma=+1\left(P, f, \omega, \rho, A_{2}, \ldots\right)$ or $\sigma P=-1, G(-1)^{I} \sigma=+1(\pi, \eta, \ldots)$ if not more than one of them has $I=1$. For any pair of such reggeons $\sigma=\sigma_{1} \cdot \sigma_{2}, I=I_{1}+I_{2}$, $G=G_{1} \cdot G_{2}$ and $G(-1)^{I} \sigma=G_{1}(-1)^{I_{1}} \sigma_{1} \cdot G_{2}(-1)^{I_{2}} \sigma_{2}=+1$. A situation is different if both reggeons have $I_{i}=1$ and add to the total isospin $I=1$. In this case $I=I_{1}+I_{2}-1$ and the product $G(-1)^{I} \sigma=-1$. So the leading cuts contributions to the small- $x$ behaviour of $g_{1}$ is given by $\rho A_{2}, \rho \pi(I=1, G=-1)$ cuts. In the following we will estimate these contributions in a simple model of a meson cloud and will show that these contributions (especially the $\pi \rho$ one) can be important in the preasymptotic region $10^{-2} \leq x \leq 0.1$.

Let us emphasize here that this classification of leading $j$-plane singularities is valid in the leading (in $1 / x$ ) approximation and contributions damped by extra $x$-factor do not satisfy to these selection rules. For example gluonic ladders can contribute to $g_{1}$ with $I=0$ at small $x$. In the following we will consider only the leading contributions for the $I=1$.

\section{The meson cloud model}

It is known for a long time that $\pi$ exchange plays an important role in the dynamics of peripheral inelastic interactions of hadrons (for review see, for example, refs. 19 [21]). In a series of papers [19], [20], [22], [4], [6] the model with "effective" reggeized pion

2 Numerically this contribution is probably very small, as it is necessary to have at least three Pomerons, and it is proportional to small spin-flip couplings of the Pomeron. 
exchange, which takes into account a contribution of other exchanges $\left(\rho, A_{2}, \ldots\right)$, has been developed and applied to a broad class of exclusive and inclusive processes in hadronic interactions. It was demonstrated that the model reproduces many characteristic features of these processes over a wide region of energy. We mention here two qualitative effects which are relevant to this subject.

It has been shown [22] that the model leads to the nontrivial behaviour of spindependent residues for the leading Regge poles, - small flip-nonflip ratio for $I=0$ reggeons $(P, f)$ and large one for $I=1$ exchange ( $\rho$ reggeon), in an agreement with experimental observations. Note that the specific spin and isospin structure of the bottom blob in Fig.1 due to pion quantum numbers plays a crucial role in description of observed spin structure. There is a strong cancellations between contributions of the nucleon and $\Delta$ isobar intermediate states for particular amplitudes.

Another example is related to the flavour structure of the quark sea in the nucleon mentioned in Introduction. It was first noted by Sullivan [23] that $\pi$ exchange can contribute also to the deep inelastic scattering (DIS) (Fig.1). In refs. [4], [6] the effective pion exchange model has been applied to calculation of antiquark distributions in a nucleon, using the distribution of $\bar{q}$ in the pion. It was noted (see also ref. [5]) that in this model $\bar{d}$ and $\bar{u}$ distributions in a nucleon are generally different. This difference is connected with the relative contributions of nucleon and $\Delta$ isobar in an intermediate state.

The distribution of antiquarks in the nucleon in this model has a simple probabilistic interpretation and can be written as a convolution of the probability $w_{\pi^{k} / N}$ to find a pion $\pi^{k}$ in a nucleon $N$ with the probability $\bar{q}_{\pi^{k}}$ to find an antiquark in the pion, summed over all types of pions [四], [6]:

$$
\bar{q}_{N}(x)=\sum_{k} \int_{x}^{1} \frac{d x_{\pi}}{x_{\pi}} w_{\pi^{k} / N}\left(x_{\pi}\right) \bar{q}_{\pi^{k}}\left(x / x_{\pi}\right) .
$$

The probability $w_{\pi^{k} / N}\left(x_{\pi}\right)$ is a sum of probabilities $w_{k}^{(R)}\left(x_{\pi}\right)$ of production of a given state $R=N, \Delta$. The latter ones expressed in terms of known $\pi N N, \pi N \Delta$ couplings and 
form factors $G_{\pi}^{R}(t)$ which determine pion off-shell dependences

$$
w_{k}^{(R)}\left(x_{\pi}\right)=\frac{x_{\pi}}{16 \pi^{2}} \int_{-\infty}^{\tau_{R}\left(x_{\pi}\right)} g_{\pi^{k} N R}^{2}(t)\left(G_{\pi}^{R}(t)\right)^{2} \quad, \quad(R=N, \Delta)
$$

Here $\tau_{R}$ is a minimal momentum transfer to the baryon $R$,

$$
\tau_{R}\left(x_{\pi}\right) \approx-\frac{m_{R}^{2} x_{\pi}}{1-x_{\pi}}+m_{N}^{2} x_{\pi}
$$

and $t$ is connected with $\tau_{R}$ and transverse momentum of produced baryon $\mathbf{k}_{\perp}$ as

$$
t \approx \tau_{R}-\frac{\mathbf{k}_{\perp}^{2}}{1-x_{\pi}} .
$$

The functions $g_{\pi^{k} N R}(t)$ contain beside corresponding coupling constants also traces over baryon indices providing extra $t$ dependence of the bottom blob.

These formulas correspond to the simple pion cloud model, - there is a cloud of pions in a fast moving nucleon and a virtual photon interacts with an antiquark (quark) of the pion. In this formulation the pion is an "effective" one and other exchanges are taken into account by a proper choice of "form factors" $G_{R}(t)$. A formulation of the meson cloud model in terms of light-cone wave functions of a pions (and other pseudoscalar and vector mesons) in a nucleon 3 was used by several authors [24]-28], [13], [29]-31], 21] and has been applied to different aspects of DIS, including the "spin crisis" [13], 31]. However the main attention was paid to a calculation of the function $g_{1}\left(x, Q^{2}\right)$ resulting not from the meson cloud but from the "core" baryon, because the diagrams of Fig.1 do not contribute to $g_{1}\left(x, Q^{2}\right)$. It has been shown [13] that the existence of the meson cloud

\footnotetext{
${ }^{3}$ It is worth to emphasize at this place that in contrast to the light-cone approach we do not consider mesonic cloud in terms of relativistic wave function. It means that interactions with the meson and the "bare" nucleon (or $\Delta$-isobar) in Figs.3a,b are not considered as interactions with the two components of the physical nucleon. We treat these diagrams as Reggeon exchanges, and the main preference of the pion exchange is connected to the smallness of the pion mass and therefore to the close position of the corresponding pole to the physical region. The $t$-dependent residue of the Regge-pole amplitude can be interpreted as a "form factor" function describing off-shell behaviour of pionic amplitudes. In the relativistic wave-function approach mesonic couplings are described in terms of $x, p_{\perp}$ dependence of the wave function, and as a result one has exponential damping for $x_{\pi} \rightarrow 0$ where pion exchange dominance is well justified and confirmed by experiments.
} 
leads to a substantional modification of the polarized quark contributions in the region $x \sim 1$ (mainly due to renormalization of the nucleon wave function), but the interaction with the core baryon (the diagrams of Fig.3b) does not influence the polarization of antiquarks from the nucleon sea.

The experience of dealing with the pion exchange model has shown that, if the pion exchange is not forbidden for a particular process, it dominates. It is related to the smallness of the pionic mass and therefore to the close position of the corresponding pole to the physical region of the process. Unluckily, as it was shown in section 2 , the $\pi \pi$ exchange does not contribute to the amplitude connected to the $g_{1}$ structure function. Here we would like to consider a new contribution of the diagram of Fig.2. It contains $\pi \rho$ exchange in the $t$-channel and corresponds to an interference between $\pi$ and $\rho$ exchanges According to a general classification given in section 2 such a state can contribute to the small -x limit of function $g_{1}$ with the total isospin $I=1$ in the $t$-channel. This means that its contributions to $g_{1}$ of proton and neutron have different signs. The new element of this model is a strong polarization of a nucleon sea and, in particular, of antiquarks of the nucleon. We will show below that this polarization is strongly flavour dependent.

Let us note here that the model gives a nonperturbative input for initial condition of QCD-evolution. So its predictions should be valid at values of $Q^{2} \sim 1 \mathrm{GeV}^{2}$ and will be modified by perturbative effects at much larger $Q^{2}$.

\section{Description of the model}

Let us consider the diagrams of Fig.4 to estimate the $\pi \rho$-exchange contribution to the $g_{1}$ structure function.

As we are interested in the imaginary part of the amplitude, particles in the intermediate state are on the mass shell. In order to get the partonic interpretation for the contribution of these diagrams it is convenient to use the light-cone variables for momenta

\footnotetext{
${ }^{4}$ Note that since our parameterization of the pion exchange contains partly the $A_{2}$ contribution we take into account effectively also the $A_{2}-\rho$ cut.
} 
in the top part of the diagram and to choose a Lorentz frame with zero 'plus'-component of the photon momentum, $q^{+} \equiv q_{0}+q_{z}=0$. This choice allows us to avoid non-partonic contributions [32]. In the Lorentz frame, where the proton is moving along the $z$ axis, one has simple kinematic relations

$$
S_{\perp}^{+}=0, \quad 2(P \cdot q)=P^{+} q^{-}
$$

and the tensor $W_{\mu \nu}$ from Eq.(1) is determined at $\mu=x$ and $\nu=y$ by only the $g_{1}$ structure function:

$$
W_{x y}^{(A)}=4 g_{1}\left(x, Q^{2}\right), \quad\left(\text { for } q_{+}=0\right) .
$$

A standard consideration of the upper blob (see e.g. [17]) reduces the answer after antisymmetrization in the photon polarization indices $\mu, \nu$ to the calculation of the matrix element for the axial current component $A^{+}=\gamma_{5} \gamma_{+}$between pion and $\rho$ meson states. The off-shell part of the quark propagator (the 'instantaneous' component 32]) is proportional to $\gamma^{+}=\gamma_{0}+\gamma_{3}$ and, since $\gamma_{+}^{2}=0$, it is possible for the 'plus'-component of the current to consider the struck quark as being on the mass shell as it is supposed in the partonic picture. This prescription corresponds to the light-front formalism 32].

Since axial curent $A^{+}$measures the helicity of the (on-shell) quark or antiquark:

$$
\bar{u}_{p, \lambda} \gamma^{+} \gamma_{5} u_{p, \lambda}=-\bar{v}_{p, \lambda} \gamma^{+} \gamma_{5} v_{p, \lambda}=4 \lambda p^{+}
$$

the result is expressed through the difference of contributions for quarks of opposite helicities, i.e. doubled contribution to the $\pi \rho$-interference term from the quark with positive helicity as it is shown schematically in Fig.5.

For the whole diagram one gets the well known relation, which connects the $g_{1}(x)$ structure function and the difference of the polarized quark distributions $\Delta q_{N}(x)$ :

$$
\begin{aligned}
& g_{1}(x)=\frac{1}{2} \sum_{q} e_{q}^{2} \Delta q_{N}(x), \\
& \Delta q_{N}(x)=q_{N}^{+}(x)-q_{N}^{-}(x)-\bar{q}_{N}^{+}(x)+\bar{q}_{N}^{-}(x),
\end{aligned}
$$

and the part of the difference due to $\pi \rho$ interference has the form similar to Eq.(5) :

$$
\Delta q_{N}(x)=\sum_{k} \int_{x}^{1} \frac{d x_{\pi}}{x_{\pi}} w_{\pi \rho}^{\alpha}\left(x_{\pi}\right) \Delta q_{\pi \rho}^{\alpha}\left(x / x_{\pi}\right) .
$$


Here $\alpha$ is a Lorentz vector index of the exchanged $\rho$-meson (we omit indices specifying charges of $\pi, \rho$ mesons), $w_{\pi \rho}^{\alpha}$ includes in analogy with Eq.(6) the contribution $B_{\pi \rho}^{\alpha}(t)$ from the bottom (nucleon) blob of the diagram of Fig.4 for positive helicity of the initial nucleon and meson "form factors" $G_{\pi, \rho}^{R}(t)$ (propagators and off-shell factors):

$$
w_{\pi \rho}^{\alpha}\left(x_{\pi}\right)=\frac{x_{\pi}}{16 \pi^{2}} \sum_{R=N, \Delta} \int_{-\infty}^{\tau_{R}\left(x_{\pi}\right)} B_{\pi \rho}^{\alpha}(t) G_{\pi}^{R}(t) G_{\rho}^{R}(t)
$$

and $\Delta q_{\pi \rho}^{\alpha}$ is a difference of polarized quark contributions coming from the top (quark) blob.

Let us discuss now separately the main ingredients entering Eqs.(12), (13).

(i) The quark blob, $\Delta q_{\pi \rho}^{\alpha}$

It is convenient to write down the part coming from the upper blob in terms of internal quark variables of the meson state $x_{q}, \mathbf{q}_{\perp}$, which are related to the variables $x, \mathbf{p}_{\perp}$ of the struck quark through the relations

$$
x_{q}=x / x_{\pi} \quad, \quad \mathbf{p}_{1 \perp}=x \mathbf{k}_{\perp}+\mathbf{q}_{\perp} .
$$

It will be assumed in the following that the nonpolarized quark distributions are the same for the $\pi$ and $\rho$ mesons:

$$
f_{q / \pi}\left(x_{q}, \mathbf{p}_{\mathbf{q} \mathbf{T}}\right)=f_{q / \rho}\left(x_{q}, \mathbf{p}_{\mathbf{q} \mathbf{T}}\right)=f_{q}\left(x_{q}, \mathbf{p}_{\mathbf{q} \mathbf{T}}\right)
$$

with the normalization condition

$$
\int_{0}^{1} d x_{q} d^{2} \mathbf{q}_{\perp} f_{q}\left(x_{q}\right)=1
$$

Then

$$
\Delta q_{\pi \rho}^{\alpha}\left(x_{q}\right)=\left(N_{\pi} N_{\rho}\right)^{-1 / 2} \int d^{2} q_{\perp} f_{q}\left(x_{q}, \mathbf{p}_{\mathbf{q} \mathbf{T}}\right) N^{\alpha}
$$

Here $N_{\pi}, N_{\rho}$ are normalization functions and the function $N^{\alpha}$ contains spin-dependent contribution corresponding to the non-diagonal $\pi \rho$ and $\rho \pi$ transition:

$$
\begin{gathered}
N^{\alpha}=N_{\pi \rho}^{\alpha}+N_{\rho \pi}^{\alpha} \\
N_{\pi \rho}^{\alpha}=\operatorname{Tr}\left[\left(\hat{p}_{1}+m_{q}\right) \frac{1-\gamma_{5} \hat{s}_{1}}{2} \Gamma^{(\pi)}\left(-\hat{p}_{2}+m_{q}\right) \Gamma_{\alpha}^{(\rho)}\right],
\end{gathered}
$$


( $s_{1}$ is a spin vector of the quark struck by the photon), and similarly (with interchange of $\pi$ and $\rho$ vertices) for $N_{\rho \pi}^{\alpha}$.

It is important that $p_{1}$ and $p_{2}$ in the equation above are on-mass-shell 4 -momenta of the quarks, $p_{1}^{2}=p_{2}^{2}=m_{q}^{2}$, and the 'minus' component of momentum is not conserved in the meson-quark-quark vertices: $p_{1}^{-}+p_{2}^{-} \neq k^{-}$. (These prescriptions have been accepted just at the point where relations (10) have been used). As a result the following kinematic relation is valid:

$$
\left(p_{1}+p_{2}\right)^{2}=M_{0}^{2}=\frac{\mathbf{p}_{q T}^{2}+m_{q}^{2}}{x(1-x)},
$$

where $M_{0}$ is a standard variable in the light-cone formalism.

The spin structure of the meson-quark vertices has been chosen as

$$
\Gamma^{(\pi)}=\gamma_{5}, \quad \Gamma_{\alpha}^{(\rho)}=\gamma_{\alpha}
$$

The normalization functions $N_{\pi}, N_{\rho}$ can be obtained e.g. by consideration of the diagonal electromagnetic form factors at zero momentum transfer. This gives

$$
\begin{aligned}
& N_{\pi}=\operatorname{Tr}\left[\left(\hat{p_{1}}+m_{q}\right) \gamma_{5}\left(-\hat{p_{2}}+m_{q}\right) \gamma_{5}\right], \\
& N_{\rho}=\frac{1}{3} \operatorname{Tr}\left[\left(\hat{p_{1}}+m_{q}\right) \gamma_{\alpha}\left(-\hat{p_{2}}+m_{q}\right) \gamma_{\alpha}\right],
\end{aligned}
$$

for the diagonal $\pi \pi$ and $\rho \rho$ transitions correspondingly. The quark mass $m_{q}$ was taken as $m_{q}=0.3 \mathrm{GeV}$ for calculations. It was pointed out above that we estimate the large distance contribution of the meson cloud to the function $g_{1}$, so the mass of the quark in our model should be considered as a constutuent quark mass and we take for it the value $m_{q}=0.3 \mathrm{GeV}$.

To show an important role of the choice of the distribution $f_{q}\left(x_{q}\right)$ we tried two extreme cases - a simple wave function description which assumes quark-antiquark content of the pion [33] and the Glück-Reya-Stratmann parameterization [34] which has a singularity at $x=0$. The parameterizations used for calculations are listed in the Appendix.

\section{(ii) The nucleon blob, $B_{\alpha}$}

The bottom blob with $R=N, \Delta$ in the intermediate state contains the nucleon traces

$$
B^{\alpha}=\sum_{R}\left(B_{\pi \rho}^{(R) \alpha}+B_{\rho \pi}^{(R) \alpha}\right)
$$


where

$$
\begin{aligned}
& B_{\pi \rho}^{(N) \alpha}=\operatorname{Tr}\left[\left(\hat{P}+m_{N}\right) \frac{1+\hat{S} \gamma_{5}}{2} \Gamma_{\pi N N} D^{(N)}(P-K) \Gamma_{\rho N N}^{\alpha}\right] \\
& B_{\pi \rho}^{(\Delta) \alpha \tau}=\operatorname{Tr}\left[\left(\hat{P}+m_{N}\right) \frac{1+\hat{S} \gamma_{5}}{2} \Gamma_{\pi N \Delta}^{\tau} D_{\tau \sigma}^{(\Delta)}(P-K) \Gamma_{\rho N N}^{\alpha \sigma}\right]
\end{aligned}
$$

The structure of the vertices $\Gamma_{\pi N R}$ and $\Gamma_{\rho N R}$ and propagators $D^{(R)}$ is given in Appendix.

Let us note that for unpolarized quark sea the contribution to the bottom blob due to nucleon and $\Delta$ isobar in the intermediate state are comparable in the magnitude. The particular values of coupling constants lead to larger values for the nucleon contribution and as a result the flavour asymmetry of antiquark sea of the proton in favour of $\bar{d}$ antiquarks. It can be shown that for the polarized quarks the contribution of $\Delta$ isobar is strongly suppressed due to spin structure of vertices. Moreover, due to $C$-parity limitation only exchanges of charged $\pi, \rho$ mesons are allowed. It results in much more drastic flavour asymmetry for polarized quark sea compared to the case of unpolarized sea.

(iii) The form factors, $G_{\pi, \rho}^{R}(t)$

We used the same $\pi$ meson form factor $G_{\pi}^{R}(t)$ as in refs. [4], [6] which allowed to give a consistent description of nucleon and $\Delta$ inclusive spectra, data on Drell-Yan process, and some other inclusive and exclusive processes in the framework of the pion exchange model. The function $G_{\rho}^{R}$ describing the off-shell behaviour of the $\rho$ meson propagator is known much worse ๆ. We used the simple exponential damping. The formulas for the form factors are given in the Appendix.

\section{Comparison with experiment}

The model formulated above allows us to calculate sea-quark polarization to the structure function $g_{1}$ of the nucleon with $I=1$. We have not tried to calculate a nonperturbative contribution to the function $g_{1}$ with $I=0$ (for calculation of $\rho-\rho$ exchange to this function see ref. [35]). Experimental data show that at small $x \sim 10^{-2}$

\footnotetext{
${ }^{5}$ We have not used the reggeized version for the off-shell behaviour of the $\rho$ exchange as it is difficult to find one which would be valid also for not small values of $x_{\rho}$.
} 
the amplitude with $I=1$ in the $t$-channel gives the main contribution. In the following we will compare our results with the $g_{1}$ of the neutron, because in this case a contribution of the valence quarks is small and contribution of the sea can be more easily separated compared to the proton case. The results for the $g_{1}^{p}$ are just opposite in sign due to $I=1$ of the considered contribution. Note that gluons (and in particular the gluon anomaly [17]) do not contribute to this isospin combination. These contributions can be important for $I=0$ amplitude.

Our model depends on several parameters which are not quite well known (e.g. $g_{\rho N N}$ coupling constant) and on assumptions listed above. So we do not pretend for detailed descriptions of the $\mathrm{I}=1$ sea component of the structure function $g 1$ but rather for a qualitative effect and demonstrate that this effect is of right order of magnitude.

In Fig. 6 the contribution of the simplest diagrams of Fig. 2 with only $q \bar{q}$ component of the pion ( $\rho$-meson) wave function is shown. Due to a fast decrease of the wave functions as $x_{q} \rightarrow 0 g_{1}$ decreases for $x<5 \cdot 10^{-2}$. It is known, however, that the valence quark distributions in the pion are substantially different (especially in the region of small $x_{q}$ ) from the behaviour corresponding to the simplest $q \bar{q}$ wave function. For a distribution of valence quarks given by GRS model [34] we obtain theoretical curves shown in Fig.7, which are in a good agreement with experimental data. Thus the model gives a reasonable estimate for both the size and the form of the sea-quark polarization in the nucleon. A word of caution concerning the above estimate of the valence quarks in the pion. The small- $x$ behaviour of the valence quarks of the pion in the GRS model $\sim 1 / x^{\gamma}$ with $\gamma \approx 0.5$ is strictly speaking true for a "diagonal" transition with quantum numbers of $A_{2}, f$ in the $t$-channel. In our case we should know the behaviour of the analogous quantity with axial quantum numbers in the $t$-channel. In the model [36], where corresponding reggeons are generated due to gluon emissions in the box diagram (Fig.8) it is possible to show that a difference between vector and axial vector contributions appears only at three-loop level (emission of two gluons in the s-channel of Fig.8). So this difference should be important in the upper blob only at small $x_{q}<x_{q}^{c r} \approx 0.1$. This means that our estimate with GRV structure function of the pion can be valid up to $x \sim \bar{x}_{\pi} x_{q}^{c r} \sim 0.01$. At smaller values of $x$ 
we expect a gradual transition to the behaviour corresponding to the $A_{1}$ Regge exchange.

We would like to emphasize that due to a smallness of the diagram with the $\Delta$ intermediate state of Fig. 2 only the $\bar{d}$ quarks of the proton ( $\bar{u}$ of the neutron) are polarized. The following relations for polarized quark distributions in the proton and the neutron are valid in the model (here we separate quark and antiquark polarized distributions unlike the notation of Eq.(11]):

$$
\begin{array}{r}
\Delta u_{\text {sea }}^{p}(x)=\Delta d_{\text {sea }}^{n}(x)=\Delta \bar{d}^{p}(x)=\Delta \bar{u}^{n}(x) \approx 6 g_{1}^{p}(x)=-6 g_{1}^{n}(x) \\
\Delta d_{\text {sea }}^{p}(x)=\Delta u_{\text {sea }}^{n}(x)=\Delta \bar{u}^{p}(x)=\Delta \bar{d}^{n}(x) \approx 0
\end{array}
$$

Thus the model predicts even higher flavour asymmetry for polarized antiquarks than for unpolarized distributions. Present estimate of polarization for antiquarks do not contradict to limits on this quantity established from a study of semiinclusive DIS [37], especially taking into account that the hypothesis of flavour symmetry for light quarks (antiquarks) of the sea has been used in the analysis of experimental data. In principle, different flavour contributions to the polarized quark sea can be separated and model predictions can be tested if accuracy of experimental data will be improved.

The most straightforward test of our model would be a measurement of the polarization asymmetries in DIS with simultaneous registration of a nucleon or $\Delta$ in the final state. For $\Delta$ production sea polarization should be absent (or small) compared to the case of neutron production off proton (or proton from a neutron target).

Let us comment on a relevance of these results for the Bjorken [38] and Ellis-Jaffe [39] sum rules. It is clear from experimental results [10] that the region of $x<0.1$, where sea quarks are important gives a substantional contribution $(\sim 30 \%)$ to the Bjorken sum rule. This means that sea-quark component of a nucleon should be important in dynamical calculation of the axial coupling constant $g_{A}$. So all calculations of the axial couplings of baryons [31] should be reconsidered with an account of this mechanism. Another important problem for the test of the Bjorken sum rule is the evaluation of the integral of the difference $g_{1}^{p}(x)-g_{1}^{n}(x)$ in the unmeasured region of very small $x$ [10]. The simplest power-law extrapolation of existing data gives a large contribution to the integral [10]. 
Our investigation indicates that a steep increase of the sea component with $I=1$ in the structure function $g_{1}(x)$ should change at $x \sim 10^{-2}$ to a slower increase (or constant behaviour) as $x$ decreases. In this case the unmeasured small- $x$ region contributes only $0.02 \div 0.03$ to the integral.

It is known [13], [31] that an inclusion of mesonic cloud to the nucleon wave function leads to a better agreement with Ellis-Jaffe sum rule. However it is difficult to obtain a good description of experimental data on $g_{1}^{p}, g_{1}^{n}$ and to solve completely the problem of "spin crisis". We hope that situation will improve with an account of interference diagrams considered here. An interference of $K$ and $K^{*}$ exchanges will lead to some polarization of $s$ and $\bar{s}$ quarks, which can be relevant for this problem.

\section{Conclusions}

In this paper we proposed a new nonperturbative mechanism for polarization of sea quarks in a nucleon. The mechanism leads to a strong flavour dependence of the sea polarization. It can not be imitated by the diagrams of perturbative QCD. An estimate of the $\pi \rho$ interference mechanism carried out in this paper shows that it can account for an unusual behaviour of the structure function $g_{1}^{n}$ in the small- $x$ region and is important for calculations of the axial couplings of baryons.

Future experiments with polarized beam and target in DIS and hadronic interactions will allow a critical test of the proposed mechanism for the sea-quark polarization.

\section{Acknowledgments}

The authors thank B.L. Ioffe and J.H. Koch for helpful discussions. K.B. wants to gratitude

the Instituto de Ciencias Nucleares, UNAM (Mexico) and especially A. Turbiner for their kind hospitality where the present work was finished. This work was supported in part by INTAS grant 93-0079ext, NATO grant OUTR.LG 971390, RFBR grants 96-02-191184a, 96-15-96740 and 98-02-17463 and DGAPA grant IN 105296. 


\section{Appendix}

Here we give a description of the main components entering the model - quark distributions used for calculations, baryon vertices and propagators, and form factors.

\section{A. Quark distributions in meson}

In order to illustrate an effect of the choice of different parametrization we used two types of distributions. The first one supposes that mesons contain only valence quark and antiquark. This two-particle distribution was parametrized in terms of the light-cone wave function [33]:

$$
f_{q}\left(x_{q}, \mathbf{p}_{q T}\right)=\left|\psi\left(x_{q}, \mathbf{p}_{q T}\right)\right|^{2}
$$

where

$$
\psi\left(x_{q}, \mathbf{p}_{q T}\right)=\sqrt{\frac{M_{0}}{2 x_{q}\left(1-x_{q}\right)}} \phi(p),
$$

with

$$
\begin{aligned}
& M_{0}^{2}=\frac{m_{q}^{2}+\mathbf{p}_{q T}^{2}}{x_{q}\left(1-x_{q}\right)}, \\
& p=\sqrt{\mathbf{p}_{q T}^{2}+p_{z}^{2}}, \quad p_{z}=M_{0}\left(x_{q}-1 / 2\right) .
\end{aligned}
$$

The function $\psi\left(x, \mathbf{p}_{T}\right)$ is normalized as

$$
\frac{1}{16 \pi^{3}} \int d x \int d^{2} p_{T}\left|\Psi\left(x, \mathbf{p}_{T}\right)\right|^{2}=1
$$

what corresponds to the standard normalization of the function $\phi(p)$ :

$$
\frac{1}{(2 \pi)^{3}} \int d^{3} p|\phi(p)|^{2}=1
$$

We use a simple oscillator-type parametrization for the wave function $\phi(p)$ [33]:

$$
\phi(p)=\pi^{-3 / 4} \beta^{-3 / 2}(2 \pi)^{3 / 2} \exp \left(-p^{2} / 2 \beta^{2}\right)
$$

with $\beta=0.32$. The second type of distribution written under an assumption of multiparticle content of a meson was taken from the paper by Glück, Reya and Stratmann 34:

$$
f_{q}\left(x_{q}, \mathbf{p}_{q T}\right)=0.471 x_{q}^{-0.501}\left(1-x_{q}\right)^{0.367}\left(1+0.632 \sqrt{x_{q}}\right) \frac{\lambda}{\pi} \exp \left(-\lambda \mathbf{p}_{q T}^{2}\right)
$$


where the value of $\lambda$ does not effect the final result and was chosen $\lambda=1$.

\section{B. The baryon vertices}

The baryon vertices were taken in the form

$$
\begin{aligned}
& \Gamma_{\pi N N}=g_{\pi N N} \gamma_{5} \\
& \Gamma_{\rho N N}^{\alpha}=g_{\rho N N}^{(0)} \gamma_{\alpha}+\frac{g_{\rho N N}^{(1)}}{2 m_{N}} \sigma_{\alpha \beta} k_{\beta} \\
& \Gamma_{\pi N \Delta}^{\tau}=g_{\pi N \Delta} p_{N \tau} \\
& \Gamma_{\rho N \Delta}^{\alpha \tau}=g_{\rho N \Delta} \gamma_{5}\left(\hat{k} g_{\alpha \tau}-\gamma_{\alpha} k_{\tau}\right), \\
& \Gamma_{\rho N \Delta}^{\alpha \tau}=g_{\rho N \Delta} \epsilon^{\alpha \tau \delta \gamma} P_{\delta} p_{\Delta}^{\gamma}
\end{aligned}
$$

where $\alpha$ and $\tau$ are Lorentz indices of the $\rho$ and $\Delta$ correspondingly. The form of the first three vertices is unambiguious, and for the forth one we tried both the structure (B.4a) used in meson exchange models [4] and the simplest structure (B.4b) which is in an agreement with experimental data on the process $\pi p \rightarrow \pi \Delta$ [41].

Only the second term in Eq.(B.2) does contribute to the small- $x$ asymptotics of the $g_{1}(x)$. The intermediate state with $\Delta$ isobar does not contribute to the asymptotics of the $g_{1}(x)$ at all (for the both structures $(\mathrm{B} .4 \mathrm{a}),(\mathrm{B} .4 \mathrm{~b})$ ). This fact results in important predictions on flavour structure of the polarized quark sea (see section 5).

The following values of coupling constants were taken [6], [40]:

$$
\begin{aligned}
\frac{g_{\pi^{0} p p}^{2}}{4 \pi} & =14.6, \\
\frac{g_{\rho^{0} p p}^{(0) 2}}{4 \pi} & =0.84, \quad g_{\rho N N}^{(1)} / g_{\rho N N}^{(0)}=6.1, \\
\frac{g_{\pi^{-} p \Delta++}^{2}}{4 \pi} & =19 \mathrm{GeV}^{-2}, \\
\frac{g_{\rho^{-} p \Delta++}^{2}}{4 \pi} & =34.7 \mathrm{GeV} .
\end{aligned}
$$

\section{Baryon propagators}

Propagators of the nucleon and the $\Delta$-isobar have standard form of those for particles of spin $1 / 2$ and $3 / 2$ :

$$
\begin{aligned}
& D_{N}(P)=\hat{P}+m_{N} \\
& D_{\Delta}(P)=\left(\hat{P}+m_{N}\right)\left[\frac{2}{3 m_{\Delta}^{2}} P_{\alpha} P_{\tau}-g_{\alpha \tau}+\frac{1}{3} \gamma_{\alpha} \gamma_{\tau}+\frac{1}{3 m_{\Delta}}\left(\gamma_{\alpha} P_{\tau}-\gamma_{\tau} P_{\alpha}\right)\right] .
\end{aligned}
$$




\section{Form factors}

The functions $G_{\pi}^{R}(t)$ were taken in the same parameterization as in ref. [6]:

$$
\begin{aligned}
& G_{\pi}^{R}(t)=\exp \left[\left(R_{1 R}^{2}+\alpha^{\prime} \ln 1 / x_{\pi}\right)\left(t-\mu^{2}\right)\right] \times \\
& \times \begin{cases}\pi \alpha^{\prime} /\left(2 \sin \left[\pi \alpha_{\pi}^{\prime}\left(t-\mu^{2}\right) / 2\right]\right), & |t| \leq\left|T_{R}\right| \\
\left.\exp \left[R_{2 R}^{2}\left(t-T_{R}\right)\right] \pi \alpha_{\pi}^{\prime} / 2 \sin \left[\pi \alpha_{\pi}^{\prime}\left(T_{R}-\mu^{2}\right) / 2\right]\right), & |t|>\left|T_{R}\right|\end{cases}
\end{aligned}
$$

with parameter values

$$
\begin{array}{lll}
R_{1 N}=0.3 G e v^{-2}, & R_{2 N}=2.0 G e v^{-2}, & T_{N}=-0.4 G e v^{2} \\
R_{1 \Delta}=0.2 G e v^{-2}, & R_{2 \Delta}=0.74 G e v^{-2}, \quad, & T_{\Delta}=-0.7 G e v^{2}
\end{array}
$$

The values of the parameters provide continuity of the derivative of function at $t=T_{R}$. In fact, as it was mentioned, the contribution of $\Delta$-isobar is negligible and only the nucleon intermediate state contributes to the $g_{1}$ structure function.

The function $G_{\rho}^{N}(t)$ was taken in the simple form consistent with meson exchange models

$$
G_{\rho}^{N}(t)=\frac{e^{R^{2}\left(t-m_{\rho}^{2}\right)}}{t-m_{\rho}^{2}},
$$

and results of calculations with $R^{2}=0.7 \mathrm{GeV}^{-2}$ and $R^{2}=1.0 \mathrm{GeV}^{-2}$ were shown as an illustration. 


\section{References}

[1] P. Amaudruz et al, Phys. Rev. Lett. 66 (1991) 2712; M. Arneodo et al, Phys. Rev. D 50 (1994) R1

[2] A. Baldit et al, Phys. Lett. B 332 (1994) 244

[3] E.A. Hawker et al, Phys. Rev. Lett. 80 (1998) 3715

[4] G.G. Arakelyan, K.G. Boreskov, A.B. Kaidalov, Sov. J. Nucl. Phys. 33 (1981) 247

[5] A.W. Thomas, Phys. Lett. B 126 (1983) 97

[6] G.G. Arakelyan, K.G. Boreskov, preprint ITEP-50-1984, 1984; Sov. J. Nucl. Phys. $41(1985) 267$.

[7] J. Ashman et al (EMC), Phys. Rev. Lett. B 206 (1986) 364; Nucl. Phys. B 328 (1989) 1

[8] D. Adams et al (SMC), Phys. Lett. B 329 (1994) 399; B 357 (1995) 248; B 396 (1997) 338; Phys. Rev. D 56 (1997) 5330

[9] P.L. Anthony et al. (E142), Phys. Rev. Lett. 71 (1993) 959; Phys. Rev. D 54 (1996) 6620

[10] K. Abe et al (E143), Phys. Rev. Lett. 74 (1995) 346; 75 (1995) 25; Phys. Lett. B 364 (1995) 61; SLAC-PUB-7753 (1998)

[11] K. Abe et al (E154), Phys. Rev. Lett. 79(1997) 26; Phys. Lett. B 404 (1997) 377; Phys. Lett. B 405 (1997) 180;

[12] K. Ackerstaff et al (HERMES), Phys. Lett. B 404 (1997) 383.

[13] V.R. Zoller, Zeit. Phys. C53 (1992) 443; C60 (1993) 141

[14] R.L. Heimann, Nucl. Phys. B 64 (1973) 429 
[15] F.E. Close, R.G. Roberts, Phys. Lett. B 336 (1994) 257

[16] B.L. Ioffe, V.A. Khoze, L.N. Lipatov, "Hard Processes", North Holland, Amsterdam, 1984

[17] M. Anselmino, A. Efremov, E. Leader, Phys. Rep. 261 (1995) 1

[18] V.N. Gribov, Proc. of XII Intern. Conf. on High Energy Physics, Dubna, 1964, p. 394

[19] K.G. Boreskov, A.B. Kaidalov, L.A. Ponomarev, preprint ITEP-92-1973 (1973), published in Proc. of Yerevan Physics School, 1975.

[20] L.A. Ponomarev, Sov. J. Part. Nucl. 7, 70 (1976).

[21] J. Speth, A.W. Thomas, Advances in Nuclear Physics, 24, 83 (1998).

[22] K.G. Boreskov, A.A. Grigoryan, A.B. Kaidalov, I.I. Levintov, Sov. J. Nucl. Phys. 27 (1978) 432

[23] J.D. Sullivan, Phys. Rev. D 5 (1972) 1732

[24] E.M. Henley, G.A. Miller,Phys. Lett. B251 (1990) 453

[25] A. Signal, A.W. Schreiber, A.W. Thomas, Mod. Phys. Lett. A6 (1991) 271; W. Melnitchouk, A.W. Thomas, A.I. Signal, Zeit. Phys. 340 (1991) 85

[26] S. Kumano, Phys. Rev. D43 (1991) 59; 3067

[27] W.-Y.P. Hwang, J. Speth, G.E. Brown, Zeit. Phys. A339 (1991) 461

[28] E. Jenkins, A.V. Manohar, Phys. Lett. B255 (1991) 558; B259 (1991) 353

[29] P.J. Mulders, A.W. Schreiber, H. Meyer, Nucl. Phys. A549 (1992) 498

[30] A. Szczurek, H. Holtmann, Acta Phys. Pol. B24 (1993) 1833

[31] H. Holtmann, A. Szczurek, J. Speth, Nucl. Phys. A596 (1996) 631 
[32] C. Michael, F.P. Payne, Zeit. Phys. C12 (1982) 145

[33] W. Jaus, Phys. Rev. D44 (1991) 2851

[34] M. Glück, E. Reya, M. Stratmann, Zeit. Phys. C53 (1992) 651

[35] R.J. Fries, A. Schäfer, Phys. Lett. B443 (1998) 40

[36] A.A. Grigoryan, N.Ya. Ivanov, A.B. Kaidalov, Sov. J. Nucl. Phys. 36 (1982) 867

[37] B. Adeva et al (SMC), Phys.Lett. B420 (1998) 180

[38] J.B. Bjorken, Phys. Rev. 148 (1966) 1467; D1 (1970) 1376

[39] J. Ellis, R.L. Jaffe, Phys. Rev. D9 (1974) 1444; Erratum D10 (1974) 1669

[40] R. Machleidt, K. Holinde, Ch. Elster, Phys. Rep. 149 (1987) 1

[41] A.B. Kaidalov, V.I. Zakharov, Pis'ma v JETP 2 (1966) 192 


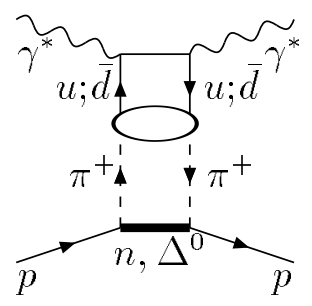

(a)

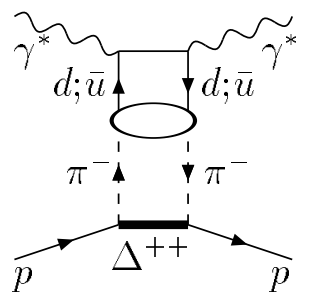

(b)

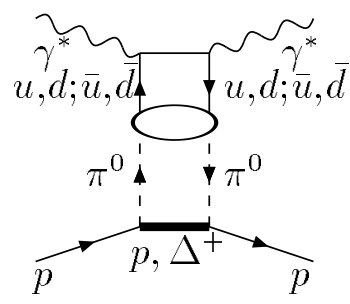

(c)

Figure 1. Pion-exchange diagrams contributing to the structure functions $F_{1}, F_{2}$

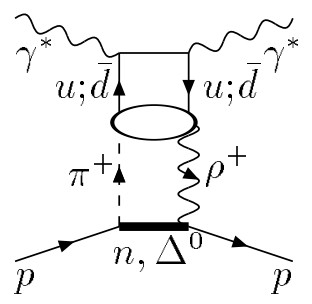

Figure 2. $\pi \rho$ interference diagram contributing to the $g_{1}$

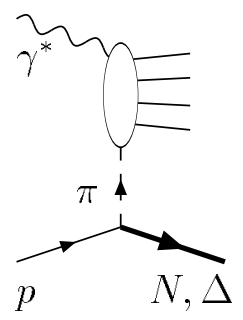

(a)

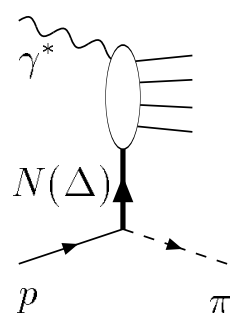

(b)

Figure 3. Scattering of a virtual photon: (a) - on a pion cloud of a nucleon and (b) - on a "core" baryon

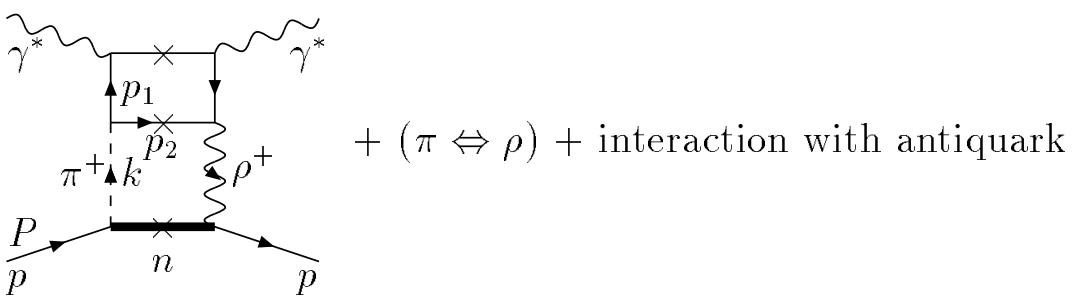

Figure 4 . The $\pi \rho$-exchange diagram with a quark box 


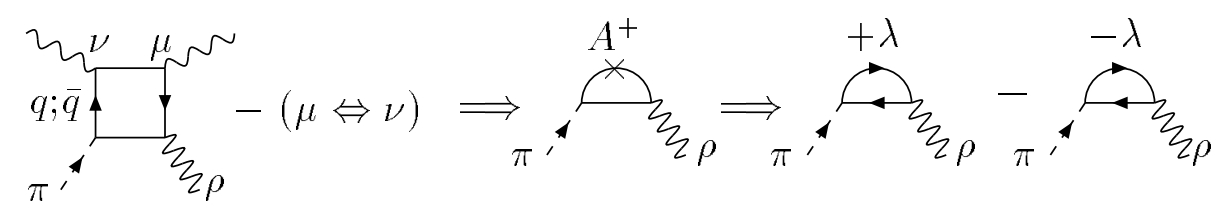

Figure 5. Schematic reduction of the $g_{1}$ amplitude to the difference of polarized quark distributions
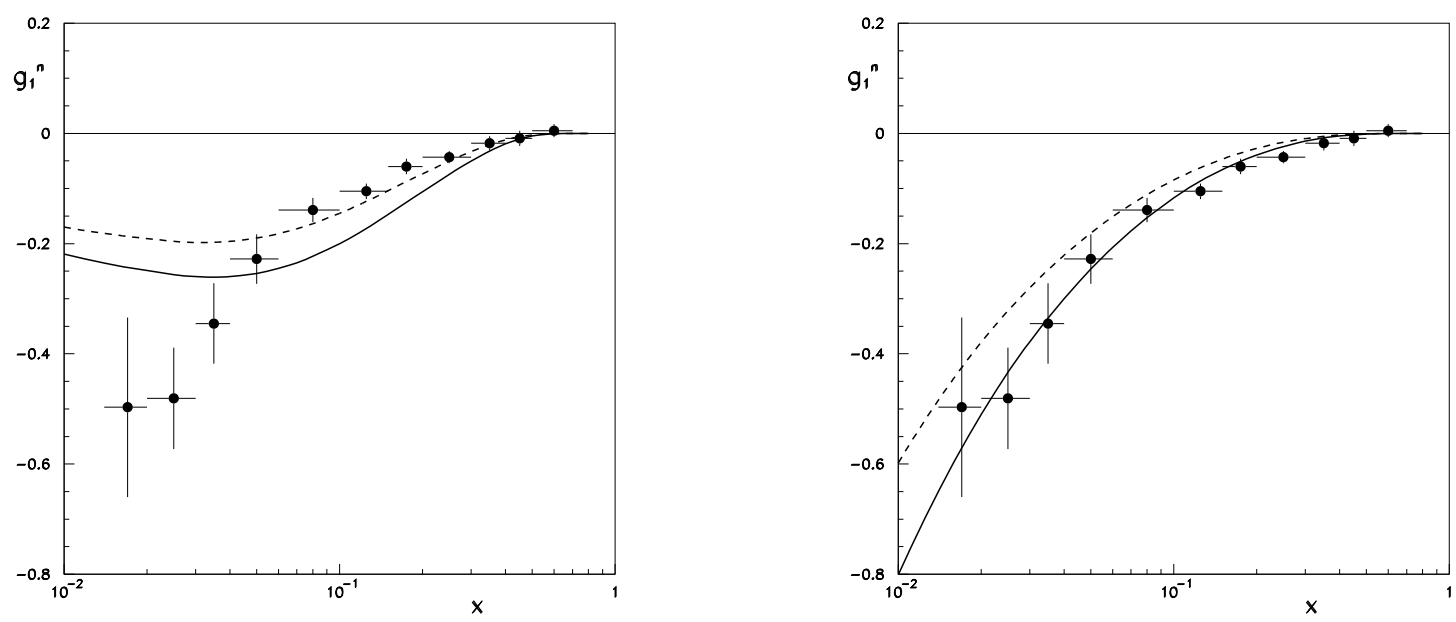

Figure 6. Description of experimental data of E154 Collaboration [11] on $g_{1}^{n}$ with $f_{q}(x)$ from the relativistic wave-function model [33] for two form-factor parameter values: $R^{2}=0.7 \mathrm{GeV}^{2}$ (solid) and $R^{2}=$ $1.0 \mathrm{GeV}^{2}$ (dashed).
Figure 7. Description of data [11] on $g_{1}^{n}$ with $f_{q}(x)$ from the GRS parametrization [34]. Solid curve corresponds to $R^{2}=0.7 \mathrm{GeV}^{2}$, and dashed one to $R^{2}=$ $1.0 \mathrm{GeV}^{2}$.

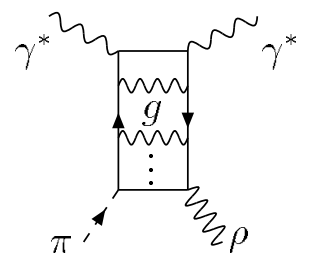

Figure 8. Gluon ladder for $\gamma^{*} \pi \rightarrow \gamma^{*} \rho$ transition 